\title{
DÉTECTION DU CHANGEMENT DE L'ÉTALEMENT URBAIN AU BAS-SAHARA ALGÉRIEN : APPORT DE LA TÉLÉDÉTECTION SPATIALE ET DES SIG. CAS DE LA VILLE DE BISKRA (ALGÉRIE)
}

\author{
Assoule Dechaicha ${ }^{1}$, Djamel Alkama² \\ 1 Département d'Architecture, Université 8 mai 1945 - Guelma. BP 401, 24000 Guelma. Algérie. \\ Tel : 213 (0) 37100553 - Fax : +213 (0) 371005 55, assoudech@gmail.com \\ 2 Département d'Architecture, Université 8 mai 1945 - Guelma. BP 401, 24000 Guelma. Algérie. \\ Tel : 213 (0) 37100553 - Fax : +213 (0) 371005 55, dj.alkama@gmail.com
}

\begin{abstract}
Résumé
L'étalement urbain constitue un des problèmes majeurs que connaissent les oasis du Bas-Sahara algérien. Le suivi et la compréhension de son évolution spatiale demeurent une étape essentielle dans toute démarche de développement soutenable de la ville oasienne. Les autorités locales ne disposent pas d'outils appropriés, susceptibles d'être mis à jour régulièrement, pour leur permettre d'agir efficacement en matière de planification et d'aménagement spatial. La télédétection spatiale et les Systèmes d'Information Géographique (SIG) offrent des opportunités permettant de surmonter ces difficultés. L'étude présentée ici consiste en l'application d'une technique de détection du changement pour cartographier et quantifier l'étalement spatiotemporel de la ville de Biskra (Algérie) durant la période 1985 - 2000, en utilisant des images Landsat des années 1985, 2000 et 2015. La comparaison post-classification des cartes d'occupation du sol a révélé une croissance des surfaces bâties, allant jusqu'au-delà des limites communales en conurbation avec l'agglomération de Chetma, au détriment d'un recul progressif de la palmeraie. Cette dernière a subi un processus de morcellement et de mitage par le bâti, conduisant à la perte de l'identité oasienne de la ville. L'objectif de notre étude est de mettre en évidence le processus de l'étalement urbain et les modifications d'occupation du sol qu'il engendre. Elle montre l'importance des méthodes de détection du changement pour la compréhension des formes d'urbanisation et l'évaluation de leurs impacts sur les écosystèmes oasiens.
\end{abstract}

Mots clés : Étalement urbain, ville oasienne, télédétection spatiale, SIG, détection du changement.

\begin{abstract}
Nowadays, urban sprawl is one of the major problems facing the Algerian Low-Sahara oases. Monitoring and understanding its spatial evolution remains an essential step for any sustainable development project of the oasis city. Local authorities do not have relevant and updated tools for effective management and spatial planning. Remote sensing and Geographic Information Systems (GIS) provide opportunities to overcome these difficulties. The present study consists in application of a change detection technique to map and quantify the spatiotemporal sprawl of Biskra City (Algeria), during the period 1985 - 2000, using three Landsat images acquired in 1985, 2000 and 2015. The post-classification comparison of land use and land cover maps has revealed a remarkable growth in the built-up areas going beyond the municipality's boundaries, in conurbation with the agglomeration of Chetma, against a progressive decline of the palm grove. It has undergone a continue process of fragmentation and conversion of its surface by the uncontrolled urbanisation, leading to the loss of the original oasis identity distinguishing this city. The main aim of this paper is to highlight the urban sprawl process and its spatial land use and land cover modifications. This study also shows the utility of change detection methods and Geographic Information Systems for monitoring the urbanisation patterns and assessing their impacts on oasis ecosystems.
\end{abstract}

Key words: Urban sprawl, oasis city, remote sensing, GIS, change detection.

\section{Introduction}

Au cours de ces trois dernières décennies, les villes du Bas-Sahara algérien ont subi un étalement spatial important. Dans un contexte de fragilité saharienne, les surfaces urbaines n'ont cessé de s'accroitre, affectant de plus en plus le paysage oasien et mettant en péril leurs écosystèmes (Ballais, 2005 ; Kouzmine, 2012). Cette modalité contemporaine de croissance est jugée génératrice de nuisances autant sur le plan environnemental que socioéconomique. L'appréhension de cette évolution rapide et non maitrisée constitue une étape essentielle pour mettre en œuvre une démarche de développement soutenable de la ville oasienne. Un meilleur suivi des mutations spatiotemporelles nécessite l'actualisation effective et permanente de l'information sur l'occupation et l'utilisation du sol. À cet égard, les collectivités locales ne disposent pas d'outils si pertinents pour maitriser et gérer l'étalement des villes oasiennes. Avec les méthodes "classiques" de relevé des états de lieu et d'enquête sur terrain, ainsi que les procédures bureaucratiques, les documents d'aménagement et d'urbanisme ne peuvent pas être mis à jour avec la régularité et la rapidité requises pour suivre l'évolution accélérée du tissu bâti (Armand, 2016).

Le recours aux approches de détection du changement (DC) permet de surmonter ces difficultés. Ces approches, basées sur la télédétection spatiale et les SIG, offrent la possibilité de suivre l'étalement rapide des surfaces urbanisées et de visualiser les transformations qu'il engendre. Les méthodes de 
détection du changement sont principalement basées sur l'analyse multitemporelle des images satellitales (Gamba et Dell'Acqua, 2016 ; Skupinski et al., 2009). Celles-ci, par leur régularité temporelle et des vues synoptiques qu'elles supportent, offrent l'opportunité d'actualiser régulièrement l'état de l'occupation et de l'utilisation du sol des territoires étudiés. Ainsi, cette ressource est devenue incontournable pour le suivi et le contrôle de l'urbanisation, notamment dans les pays en voie de développement où ce phénomène est plus accentué et la mise en évidence de ces impacts semble moins évidente (Armand, 2016 ; Dureau et Weber, 1995).

En se basant sur les images satellitales, l'objectif de cette étude est double : d'une part, mettre en évidence du processus d'étalement d'une grande ville oasienne, celle Biskra, durant la période 1985 - 2015, en essayant d'établir une cartographie de changement caractérisant son évolution spatiotemporelle; d'autre part, quantifier cette croissance spatiale afin de se rendre compte de ses répercussions sur la palmeraie. Cette étude est structurée en quatre sections. Après l'introduction, des éléments conceptuels liés à la détection du changement sont abordés. La deuxième section porte sur la présentation de l'aire d'étude et de la démarche méthodologique suivie. Les résultats obtenus ainsi que leurs interprétations sont présentés dans la troisième section. À la fin, cette étude est finalisée par des éléments de conclusion et des perspectives.

\section{La Détection du Changement pour suivre l'étalement urbain : cadre théorique}

La détection du changement d'occupation et d'utilisation des sols est l'une des principales applications de la télédétection spatiale (Mas, 2000). II s'agit d'une démarche de comparaison de scènes satellitales multi-dates. Elle consiste à mettre en valeur le changement biophysique de la couverture du sol durant une période donnée, à travers le calcul de la variation de radiance entre pixels ayant les mêmes localisations (Mas, 2000 ; Singh, 1989). Les méthodes de détection du changement s'appuient principalement sur la télédétection spatiale et les SIG pour l'acquisition, le traitement et l'exploration des données spatiotemporelles. Les SIG facilitent ainsi la modélisation et la prédiction de différents scénarios ainsi que leur évaluation. Cette approche permet d'appréhender le processus d'étalement des villes à travers l'identification des différents changements d'occupation et d'utilisation du sol s'opérant sur les territoires urbains et périurbains. Cette démarche peut aider les autorités locales à agir efficacement en matière de planification spatiale, dans une optique de développement durable des territoires avec moins d'impact sur l'environnement (Thériault et al., 2011 ; Weng, 2007). La finalité de cette approche peut inclure la localisation géographique du changement à travers une cartographie du changement, l'identification du type de changement ainsi que son évaluation quantitative et qualitative (Coppin et al., 2004 ; Hussain et al., 2013 ; Im et Jensen, 2005).

Diverses méthodes ont été développées et adoptées pour la détection du changement. Selon l'approche mise en œuvre, ces méthodes peuvent être, brièvement, répertoriées en trois catégories: (i) des méthodes algébriques, ou de comparaison directe, qui s'appuient sur le calcul de la variation radiométrique; (ii) des méthodes de comparaison après transformation et normalisation d'images (rehaussement); (iii) des méthodes post-classification basées sur la classification d'images diachroniques. Le choix méthodologique dépend généralement des objectifs escomptés, de I'hétérogénéité du territoire observé, de la résolution spatiale et temporelle des images utilisées et des connaissances de l'analyste (Hussain et al., 2013 ; D Lu et al., 2004; Mas, 2000).

\section{Matériel et méthodes}

\subsection{Aire d'étude}

La ville de Biskra (Algérie), la plus grande ville dans le Sud algérien, est située à $470 \mathrm{~km}$ au sud-est de la capitale Alger, sur les limites septentrionales du BasSahara algérien. Elle est située dans une oasis historique souvent appelée porte du désert et elle est le Chef-lieu de Wilaya, micro-capitale du pays des Ziban, pays oasien distingué par sa production agricole, particulièrement de dates. Géographiquement, elle est localisée sur les coordonnées suivantes: latitude: $34^{\circ} 51^{\prime} 00^{\prime \prime}$ Nord ; longitude : $5^{\circ} 44^{\prime} 00 "$ Est ; Altitude : $120 \mathrm{~m}$ (Bouzahzah, 2015).

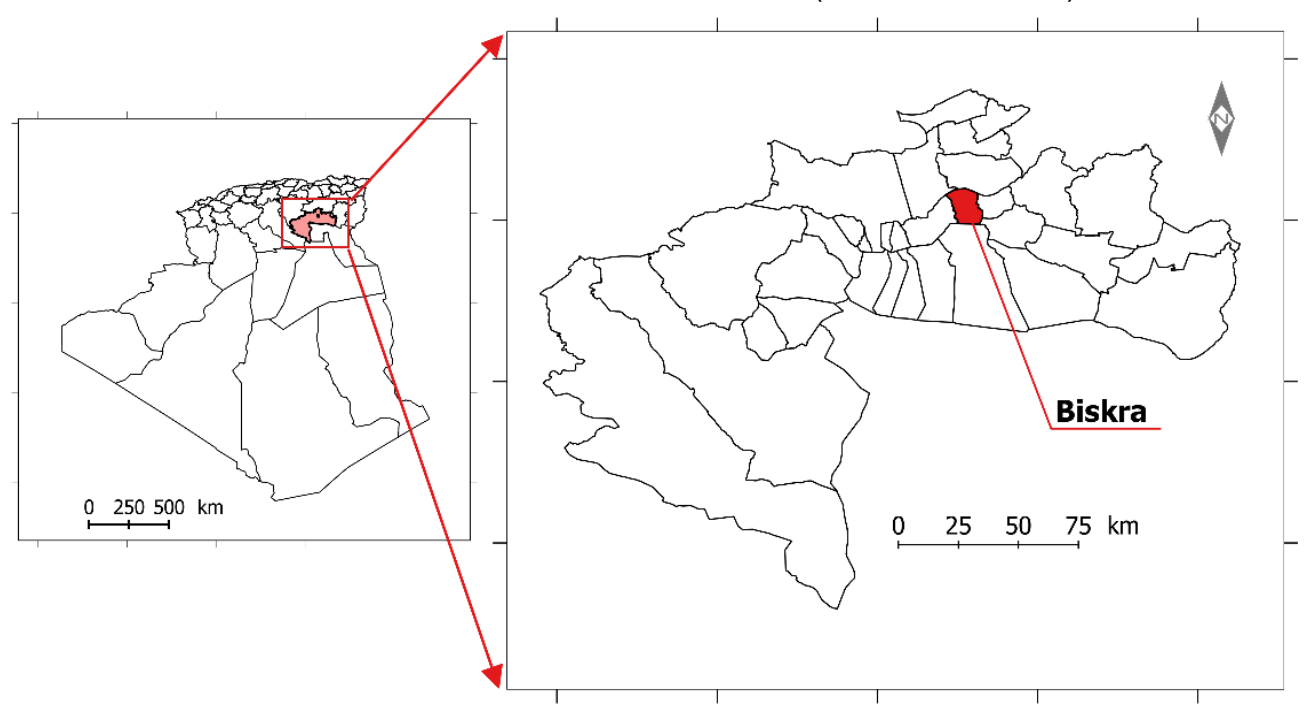

Figure 1 : Situation de l'aire d'étude.

(Établie par les auteurs à partir d'une cartographie DIVA-GIS : http://www.diva-gis.org) 
Topographiquement, la ville de Biskra est implantée sur un site appartenant à une zone de jonction entre deux domaines naturels opposés, les monts de l'atlas saharien (Monts du Zab et des Aurès, $2329 \mathrm{~m}$ ) et les plaines étendues de la frange saharienne $(60 \mathrm{~m})$. Sur une surface communale de l'ordre de $127,53 \mathrm{~km}^{2}$, les plaines argilo-sablonneuses occupent la majeure partie, avec environ $80 \%$ de l'assiette, la zone de piémont présente $10 \%$, le reste est une zone de relief couvrant la partie nord de la commune (Bouzahzah, 2015). Le climat de Biskra est un climat aride, caractérisé par des hivers froids et moins rigoureux et des étés chauds et très secs. Les températures moyennes varient entre et $5,8^{\circ} \mathrm{C}$ au mois de janvier et $40,6^{\circ} \mathrm{C}$ au mois de juillet. Les moyennes d'humidité sont relativement basses, elles varient entre $59,85 \%$ au mois de décembre et $26,42 \%$ en juillet. Les précipitations sont très rares et irrégulières avec un cumul annuel avoisinant les $140 \mathrm{~mm}$. Les vents dominants sont de deux directions : ceux du nord-est, relativement humides qui soufflent pendant l'hiver et le printemps ; ceux venant du sud et du sud-ouest (le sirocco), des vents de sable, chauds et secs, caractérisant l'été (Bouzahzah, 2015; Hanafi et Alkama, 2017). La population de Biskra a enregistré une importante évolution. Elle est passée de 52519 habitants en 1966 à 211890 habitants en 2015, une multiplication par quatre sur une période de 50 ans. Cette explosion démographique pose actuellement de nombreux problèmes comme la consommation excessive de l'espace, la congestion urbaine, les infrastructures et l'aménagement inadaptés et une perte progressive de l'identité oasienne (Farhi, 2002 ; Farhi et Hadhaga, 2018 ; Kouzmine, 2012).

\subsection{Données et matériel utilisés}

La présente étude consiste à caractériser l'évolution de la tâche urbaine de la ville de Biskra sur une période de 30 ans, entre 1985 et 2015 . Une période durant laquelle l'étalement urbain semble plus significatif. Pour ce faire, une série séquentielle constituée de trois images multispectrales Landsat a été acquise par le biais de I'USGS (United States Geological Survey). Les images acquises correspondent aux années suivantes: 1985, 2001 et 2015. Ces trois scènes satellitales sont sélectionnées en faveur de leur similarité temporelle (scènes prises pendant les mêmes saisons de l'année et à des heures très proches) afin d'assurer une meilleure similitude en matière de conditions atmosphériques et phénologiques (Coppin et al., 2004 ; Dengsheng Lu et Weng, 2007). Les caractéristiques des images obtenues sont illustrées dans le tableau 1.

\begin{tabular}{|c|l|c|c|c|c|c|}
\hline Image & \multicolumn{1}{|c|}{ Capteur } & Date & Bandes & Nébulosité & RMSE $(\mathbf{m})$ & Rés. spatiale $(\mathbf{m})$ \\
\hline Image 1 & Landsat 5 TM & $30-04-1985$ & $1-2-3-4-5-7$ & 0 & 4416 & 30 \\
\hline Image 2 & Landsat 7 ETM+ & $15-04-2000$ & $1-2-3-4-5-7$ & 0 & 4148 & 30 \\
\hline Image 3 & Landsat 8 OLI-TIRS & $03-05-2015$ & $2-3-4-5-6-7$ & 0 & 6786 & 30 \\
\hline
\end{tabular}

Tableau 1 : Caractéristiques des images Landsat utilisées

Des cartes auxiliaires de référence sont aussi utilisées. II s'agit de trois plans parcellaires représentant les états d'utilisation et d'occupation du sol des années 1986, 2002 et 2014 ; elles sont élaborées à des échelles plus fines (1/5000) par l'Institut National de Cartographie et de Télédétection (INCT). Trois images Geotiff (1985 - 2000 - 2015) fournies en couleur naturelle par I'USGS sont également utilisées. Le recours à ces images permet d'identifier les surfaces situées en dehors des périmètres urbains et non couvertes par les plans parcellaires.

La chaîne de traitement (classification et postclassification) des images satellitales est effectuée au moyen du logiciel QGIS (Congedo, 2016 ; Leroux et al., 2018).

\subsection{Démarche méthodologique}

La démarche suivie dans cette étude est structurée en quatre étapes: (i) prétraitement et normalisation des images acquises ; (ii) une procédure de classification et de post-traitement (perfectionnement) ; (iii) évaluation de la performance de la classification ; (iv) comparaison post-classification des cartes thématiques générées à l'issue de la classification (Ban et Yousif, 2016 ; Dengsheng Lu et Weng, 2007 ; Nath et al., 2014).

\subsubsection{Prétraitement et normalisation des images}

Les images sélectionnées pour cette étude (collection de niveaux L1TP) sont géométriquement autorectifiées et géocodées préalablement à leur diffusion par l'USGS, selon le système de référencement géographique WGS 84 Zone 31 Nord. L'estimation standard d'erreur RMSE (Rout Mean Square Error) a révélé un niveau négligeable d'erreur (moins de 0,23 pixel). La superposition des images est visuellement vérifiée. La zone d'étude est extraite à travers une fenêtre de découpe incluant l'actuel périmètre urbain ainsi que son environnement périurbain. Un calibrage radiométrique est ainsi effectué en exécutant une correction atmosphérique de type TOA (Top Of Atmospheric) et une soustraction des objets non attribués en appliquant le modèle DOS1 (Dark Object Subtraction) (Congedo, 2016 ; NASA, 2011 ; Tucker et al., 2004). Par la suite, un rehaussement des images est appliqué afin d'améliorer leur qualité et faciliter en même temps leur interprétation visuelle. Une composition colorée en fausses couleurs a été retenue avec la combinaison des bandes (4-3-2) pour les images TM et ETM+ et (5-4-3) pour celle de Landsat 8 (figure 2). Cette composition, après plusieurs autres, a montré de meilleures discriminations des surfaces et des objets constituant les différents types d'occupation du sol (Collet et Caloz, 2001 ; Jensen et Lulla, 1987) 

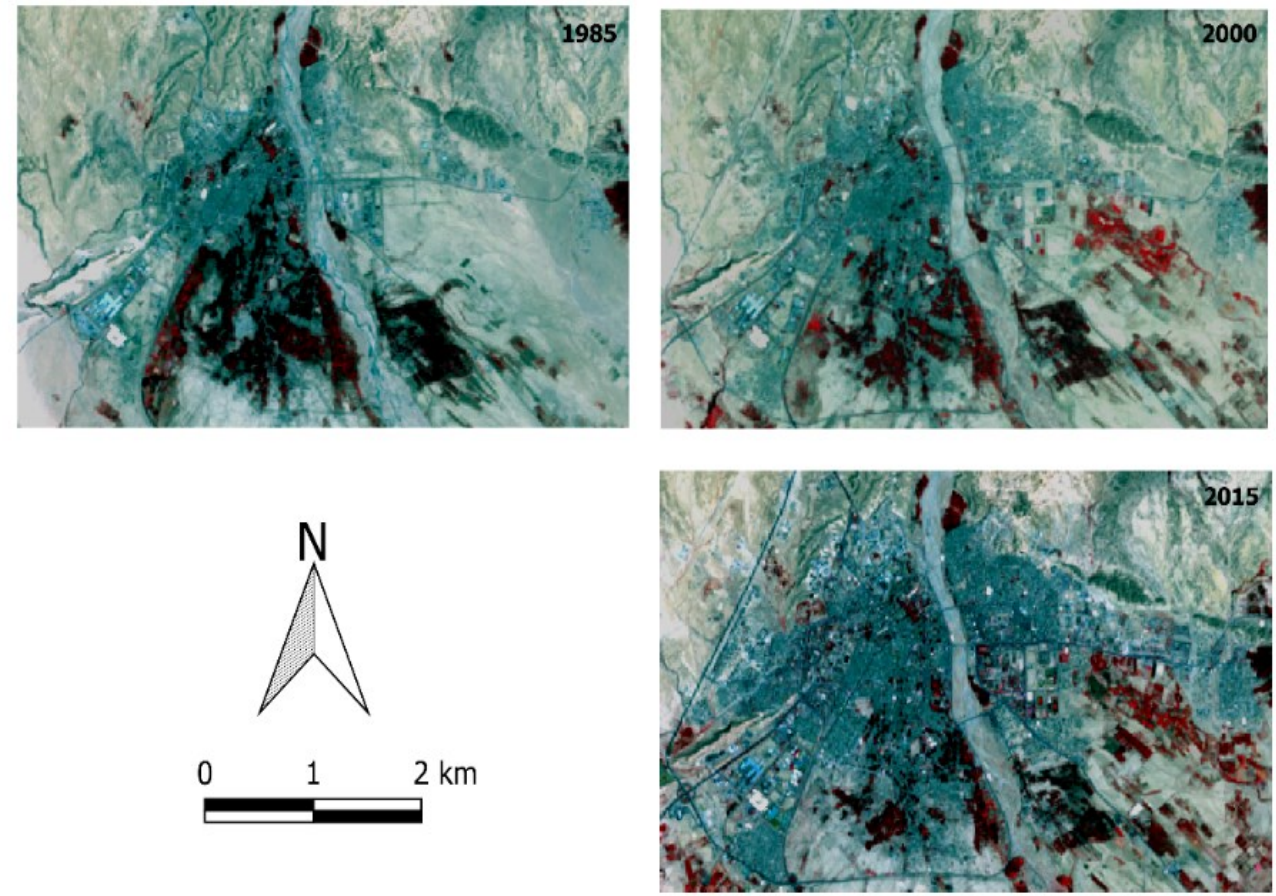

Figure 2 : Compositions en fausses couleurs des images utilisées (1985 - 2000 - 2015)

\subsubsection{Classification supervisée et post-traitement}

La classification des données satellitales est une méthode efficace permettant l'extraction de l'information spatiale. Elle consiste à attribuer à chaque pixel son appartenance en matière d'occupation du sol. Dans cette étude, une classification de type supervisée a été entamée (Girard et Girard, 2010 ; Dengsheng Lu et Weng, 2007). En se basant sur les documents de référence et la connaissance du terrain, quatre classes d'occupation du sol sont définies : surfaces urbanisées, palmeraie (haute végétation), basse végétation, et sol nu. La description de chacune de ces classes est illustrée dans le tableau 2. L'échantillonnage des zones d'entrainement (zones de test) est établi par photo- interprétation des images en compositions colorées, en correspondance avec les cartes de référence (Du et al., 2014 ; Im et Jensen, 2005 ; Munafò et Congedo, 2017). Des polygones représentatifs de différentes classes sont générés à travers l'algorithme "Region Growing » qui consiste à créer, à partir d'un pixel de départ, une région homogène incluant des pixels ayant des propriétés spectrales similaires (Congedo, 2016 ; Rajendran et Mani, 2015). L'analyse des distances spectrales a révélé quelques chevauchements, particulièrement entre la classe du sol nu et celle du bâti. Pour régler cette anomalie, nous avons multiplié les sous-classes au niveau des zones de confusion avec application de seuillage automatique permettant leurs séparabilités spectrales (Congedo, 2016 ; Sezgin et Sankur, 2004).

\begin{tabular}{|c|l|l|}
\hline $\mathbf{N}^{\circ}$ classe & \multicolumn{1}{|c|}{ Labelle } & \multicolumn{1}{c|}{ Description } \\
\hline 01 & Surface urbanisée & $\begin{array}{l}\text { Constructions résidentielles, commerciales et de services, complexes } \\
\text { touristiques et industriels, zone d'activité, voies de circulation, zones urbaines } \\
\text { mixtes. }\end{array}$ \\
\hline 02 & Palmeraie & Haute végétation (phoœniciculture). \\
\hline 03 & Basse végétation & Prairies, maraichages et sols à vocation agricole. \\
\hline
\end{tabular}

Tableau 2 : Labélisation et description des classes retenues

Une classification supervisée a été effectuée en exécutant l'algorithme "Maximum de vraisemblance » (Dengsheng Lu et Weng, 2007 ; Mather et Tso, 2016 ; Nath et al., 2014 ; Phiri et Morgenroth, 2017). Par la suite, une amélioration post-traitement des cartes générées par la classification est entamée. Elle consiste à corriger les confusions induites par la classification et l'élimination des pixels isolés en appliquant un filtre de $3 \times 3$ pixels. À la fin de cette étape, un traitement de reclassification est appliqué sur les trois cartes en vue de les rendre homogènes et comparables (Dengsheng Lu et Weng, 2007). Les sous-classes du sol nu sont regroupées en une seule classe, de même pour celles des surfaces urbanisées. Concernant la végétation, nous avons de nouveau distingué deux classes: végétation urbaine localisée à l'intérieur de l'actuel périmètre urbain, principalement représentée par la palmeraie ; végétation périurbaine au-delà du périmètre bâti, qui correspond aux nouvelles exploitations agricoles dans la périphérie de la ville. Cette reclassification permettra la visualisation de l'évolution spatiotemporelle de la palmeraie à l'intérieur de la ville séparément de celle s'opérant en périphérie. 


\subsection{3. Évaluation de la performance : la matrice de confusion}

L'évaluation de la classification est cruciale pour pouvoir procéder à l'analyse thématique. Elle renseigne sur le niveau de précision des cartes produites par la classification. II existe plusieurs méthodes d'évaluation de la classification; la plus courante est la matrice de confusion (Congalton, 1991). Un indice synthétique issu de la matrice de confusion est également utilisé dans l'évaluation de la précision. II s'agit de l'indice Kappa (Khat). C'est un indicateur de qualité servant à mesurer la performance d'une classification à travers l'examen de l'ensemble des éléments constituant la matrice (Congalton, 1991 ; Stehman, 1996). Ce mode de calcul permet l'obtention d'une évaluation fiable du niveau de précision de la classification, à la différence du premier indice qui est plus global et dont le calcul est focalisé seulement sur les éléments de la diagonale (Collet et Caloz, 2001). Pour une valeur de $K_{\text {hat }}$ supérieure ou égale à 0.8 , la classification est statistiquement jugée acceptable ; alors que si le $K_{\text {hat }}$ varie entre 0,4 et 0,8, la classification est considérée de qualité moyenne (Congalton et Green, 2008 ; Landis et Koch, 1977). Dans cette étude, l'évaluation de la précision est effectuée en procédant en premier lieu à la digitalisation de 200 points de contrôle, créés et répartis aléatoirement sur toute la zone d'étude et couvrant toutes les classes, puis, en second lieu, à l'enregistrement des données réelles à partir de la photo-interprétation des cartes de référence. La matrice de confusion est par la suite générée à partir du calcul matriciel de comparaison (SIG).

\subsubsection{Détection et calcul du changement}

Lors de cette étape, les cartes thématiques générées à l'issue de la classification sont soumises à une opération de comparaison post-classification. L'objectif de cette analyse est de visualiser et décrire le processus de l'étalement de la tâche urbaine de la ville de Biskra à travers l'identification des différents changements d'occupation du sol. Deux intervalles temporels sont nuancés : $1985-2000$ et $2000-2015$. Le résultat pour chaque période est une carte de changement complétée par un rapport descriptif. Sur la carte de changement sont localisées les surfaces inchangées et celles converties durant la période concernée; le rapport descriptif illustre quantitativement l'évolution de chaque classe en indiquant la nature de mutation (changement d'une classe à une autre).

\section{Résultats et discussion}

\subsection{Validation de la classification}

Les cartes thématiques obtenues sont illustrées dans la figure 4. Elles correspondent respectivement aux années 1985, 2000 et 2015 . Les matrices de confusion générées pour ces trois cartes ont montré un niveau satisfaisant de précision, aussi bien pour la précision globale que la précision des classes, notamment celles des surfaces urbanisées et de la palmeraie. L'indice de Kappa (Khat) a ainsi montré un niveau acceptable de précision avec les valeurs $0.81,0.89$ et 0.90 pour les années 1985, 2000 et 2015 respectivement. La synthèse de cette évaluation est illustrée dans le tableau 3.

\begin{tabular}{|l|c|c|c|}
\hline Type d'évaluation & $\mathbf{1 9 8 5}$ & $\mathbf{2 0 0 0}$ & $\mathbf{2 0 1 5}$ \\
\hline Précision globale (\%) & 91,01 & 95,18 & 94,87 \\
\hline Précision classe « surface urbanisée » (\%) & 92,20 & 94,30 & 97,85 \\
\hline Précision classe « palmeraie » (\%) & 96,00 & 92,30 & 98,10 \\
\hline Indice Khat & 0,81 & 0,89 & 0,90 \\
\hline
\end{tabular}

Tableau 3 : Précision de la classification des trois images 1985, 2000 et 2015

Il est à noter que cette évaluation a révélé quelques résultats erronés mais négligeables, correspondant à certaines confusions de classification au niveau des lisières entre le sol nu (de nature caillouteuse) et le bâti.

\subsection{La cartographie du changement : un remarquable étalement du bâti}

La détection du changement a conduit à l'obtention d'une cartographie spatiotemporelle (figure 3) illustrant l'évolution de la ville de Biskra entre 1985 et 2015. La première lecture diachronique des trois cartes montre une nette croissance des surfaces urbanisées, au détriment d'un important recul de la palmeraie. Cette dernière, positionnée en 1985 en dehors de la ville, s'est progressivement trouvée incorporée dans le périmètre urbain. Par ailleurs, la végétation périurbaine a connu une augmentation de sa surface, notamment au sud-est de la ville. 

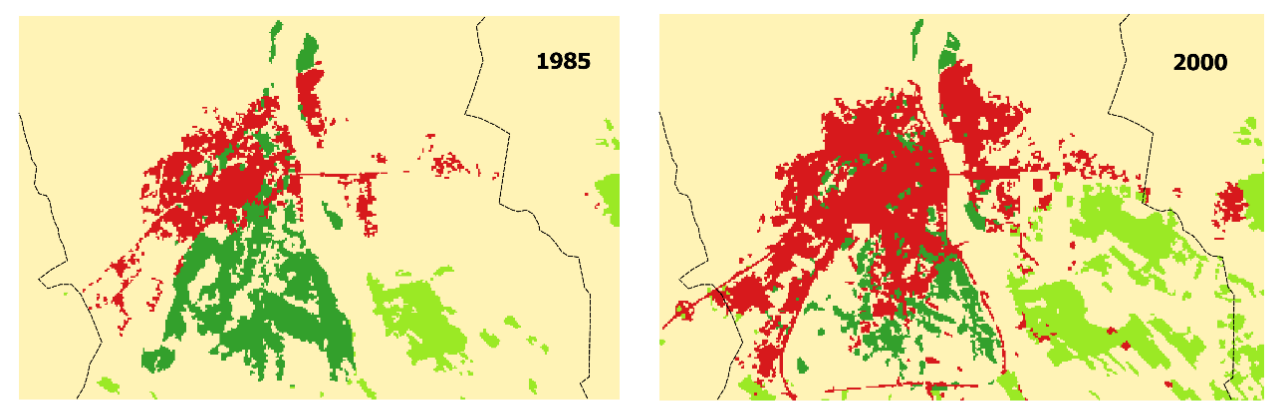

Surface urbanisée

Palmeraie

Végét. périurbaine Sol nu

--- Limites communales
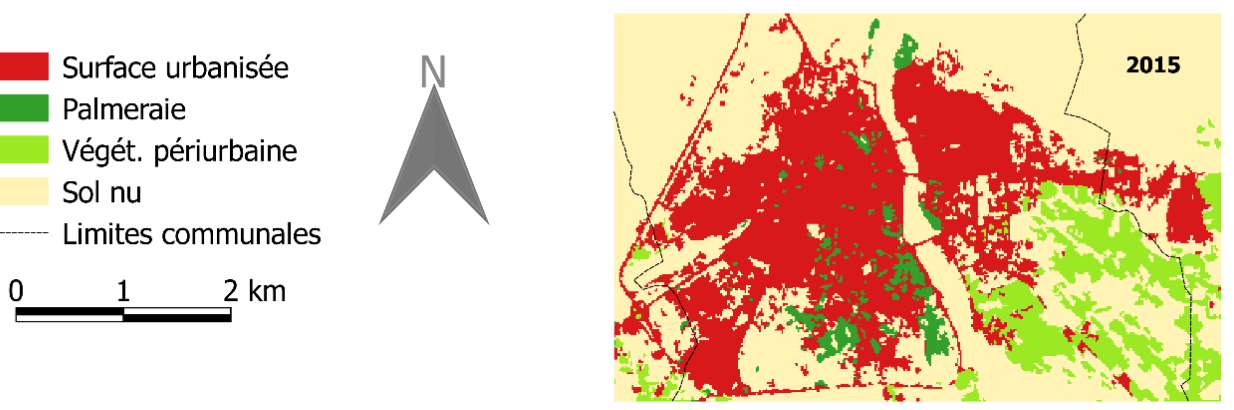

Figure 3 : Évolution spatiotemporelle des classes d'occupation du sol de l'oasis de Biskra (1985 - 2015)

Spatialement, l'extension de la tâche urbaine s'est opérée suivant trois directions: vers le sud, par empiétement des surfaces appartenant à la palmeraie; vers l'ouest et le sud-ouest, le long des axes routiers, notamment la $\mathrm{RN}^{\circ} 46$, où un début de franchissement des limites communales est remarqué sur le territoire de la commune d'El-Hadjeb ; vers l'est, au-delà de l'oued de Biskra. Le long de cette dernière direction, un processus de conurbation urbaine est observé, particulièrement durant la période 2000 - 2015, intégrant un autre territoire communal limitrophe, celui de Chetma à l'est de la ville de Biskra. En revanche, la palmeraie s'est progressivement rétrécie au profit du bâti, avec succession de deux processus : dans un premier temps, occupation des surfaces interstitielles dégradées à l'intérieur de la palmeraie, puis, et dans un second temps, conversion (mitage) de la surface cultivée.

Sur le plan quantitatif, le tableau 4 et la figure 4 illustrent cette évolution spatiale.

\begin{tabular}{|c|c|c|c|c|c|}
\hline \multirow{2}{*}{ Classe } & \multicolumn{3}{|c|}{ Surface (ha) } & \multirow{2}{*}{$\begin{array}{c}1985-2000 \\
+/- \text { (ha) }\end{array}$} & \multirow{2}{*}{$\begin{array}{c}2000-2015 \\
+/- \text { (ha) }\end{array}$} \\
\hline & 1985 & 2000 & 2015 & & \\
\hline Surface urbanisée & 488,43 & 1279,62 & 2673,00 & $+791,19$ & $+1393,38$ \\
\hline Palmeraie & 635,13 & 314,01 & 241,29 & $-321,12$ & $-72,72$ \\
\hline Végétation périurbaine & 247,50 & 673,92 & 786,42 & $+426,42$ & $+112,50$ \\
\hline Sol nu & 6772,14 & 5875,65 & 4442,49 & $-896,49$ & $-1433,16$ \\
\hline
\end{tabular}

Tableau 4 : Évolution surfacique des classes d'occupation et d'utilisation des sols

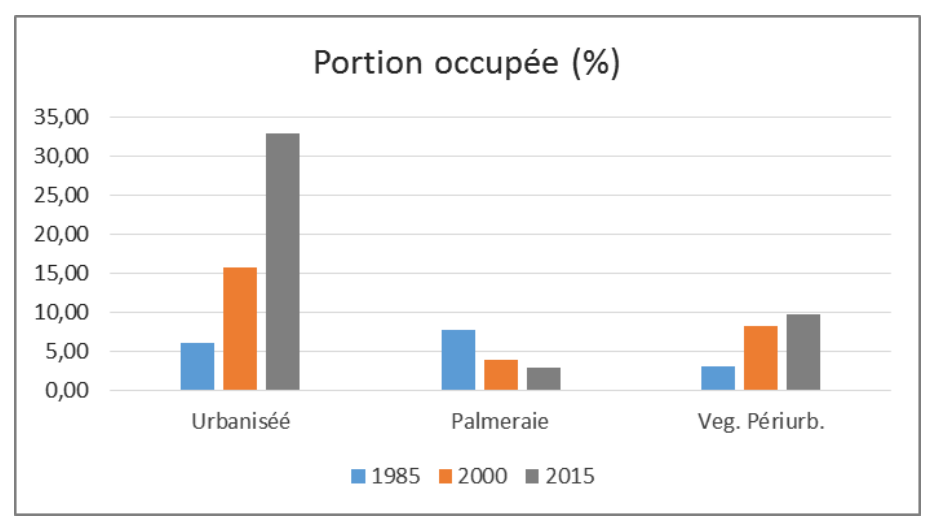

Figure 4 : Évolution des portions surfaciques des classes d'occupation du sol (1985 - 2015) 
La figure ci-dessus (figure 4) met en valeur l'ampleur de la croissance des surfaces bâties par rapport à celle de la végétation urbaine. La lecture du tableau révèle une variation de rythme de croissance entre les deux périodes $(1985-2000)$ et $(2000-2015)$.

Au cours de la première période, la classe bâtie a enregistré une croissance de sa surface. Elle est passée de 488,43 ha en 1985 pour atteindre 1279,62 ha en 2000 avec une augmentation de 791,19 ha, soit une évolution annuelle estimée à 52,75 ha. En revanche, la palmeraie a connu un processus inversé. Sa surface s'est diminuée de 635,13 ha en 1985 à 314,01 ha en 2000 avec une perte de 321,12 ha, soit $50,56 \%$ de surface perdue et 21,41 ha de perte annuelle. L'agriculture périurbaine a enregistré un gain de surface estimé à 426,42 ha. Cette classe est passée de 247,50 ha en 1985 à 673,92 ha en 2000 avec une croissance annuelle de l'ordre de 28,43 ha par an. La classe du sol nu a ainsi perdu de sa surface dont 896,49 ha converties par les autres classes. La figure 5 synthétise cette première période.
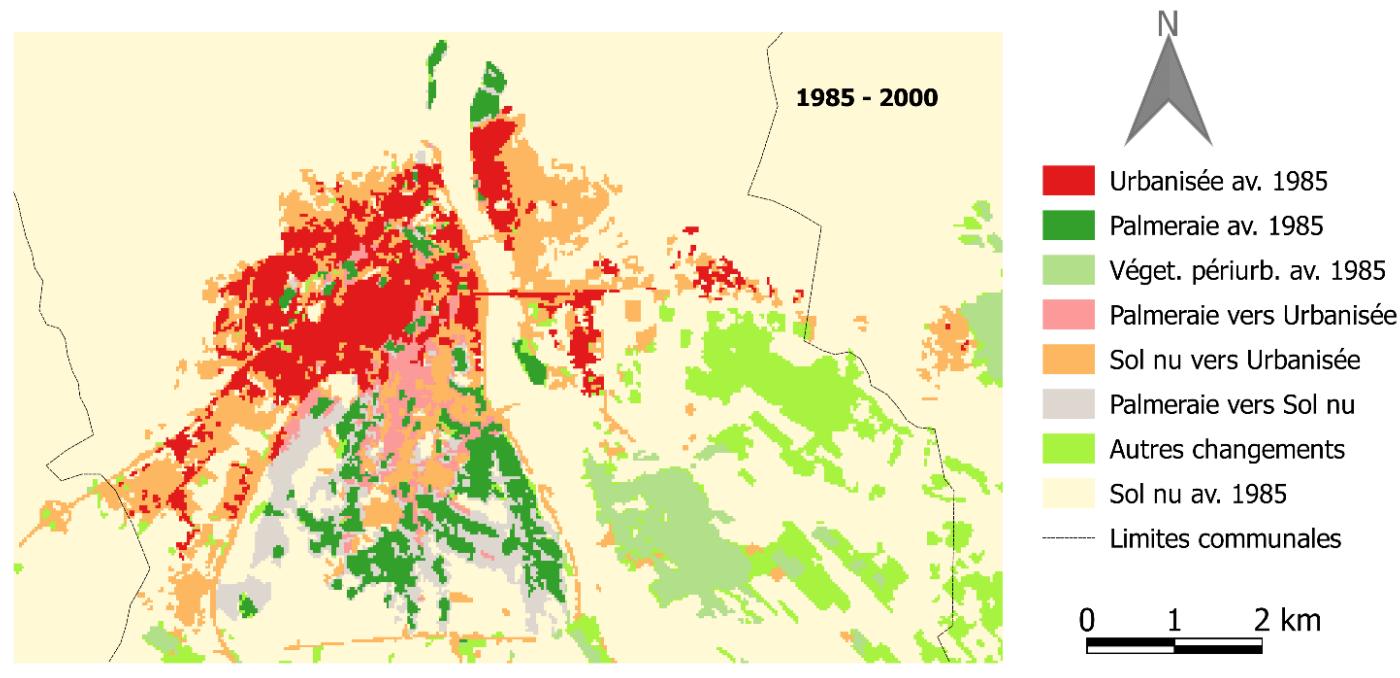

Figure 5 : Changement d'occupation du sol entre 1985 et 2000

Durant la seconde période (2000 - 2015), illustrée par la figure 6 , le changement d'occupation et d'utilisation du sol se poursuit, mais avec une variation d'ampleur. La classe du bâti a marqué une évolution plus accélérée par rapport à la première période. Elle a intégré plus de 1393,38 ha pour atteindre 2673 ha d'emprise en 2015, soit une évolution annuelle de l'ordre de 92,89 ha (contre 52,75 ha/an durant la période 1985 - 2000). Par opposition, la palmeraie a continué de perdre de sa surface, mais avec un rythme moins rapide. La surface disparue durant cette période est estimée à 72,72 ha (contre 321,12 ha entre 1985 et 2000), soit une perte de 4,85 ha par an. L'évolution de la surface occupée par la végétation périurbaine a enregistré un ralentissement par rapport à la première période. Avec une augmentation annuelle de 7,50 ha, cette classe a pu atteindre 786,42 ha en 2015 . La dernière classe, celle du sol nu, a enregistré une accélération du rythme de conversion vers les autres classes avec une moyenne annuelle estimée à 95,54 ha par an (figure 6).
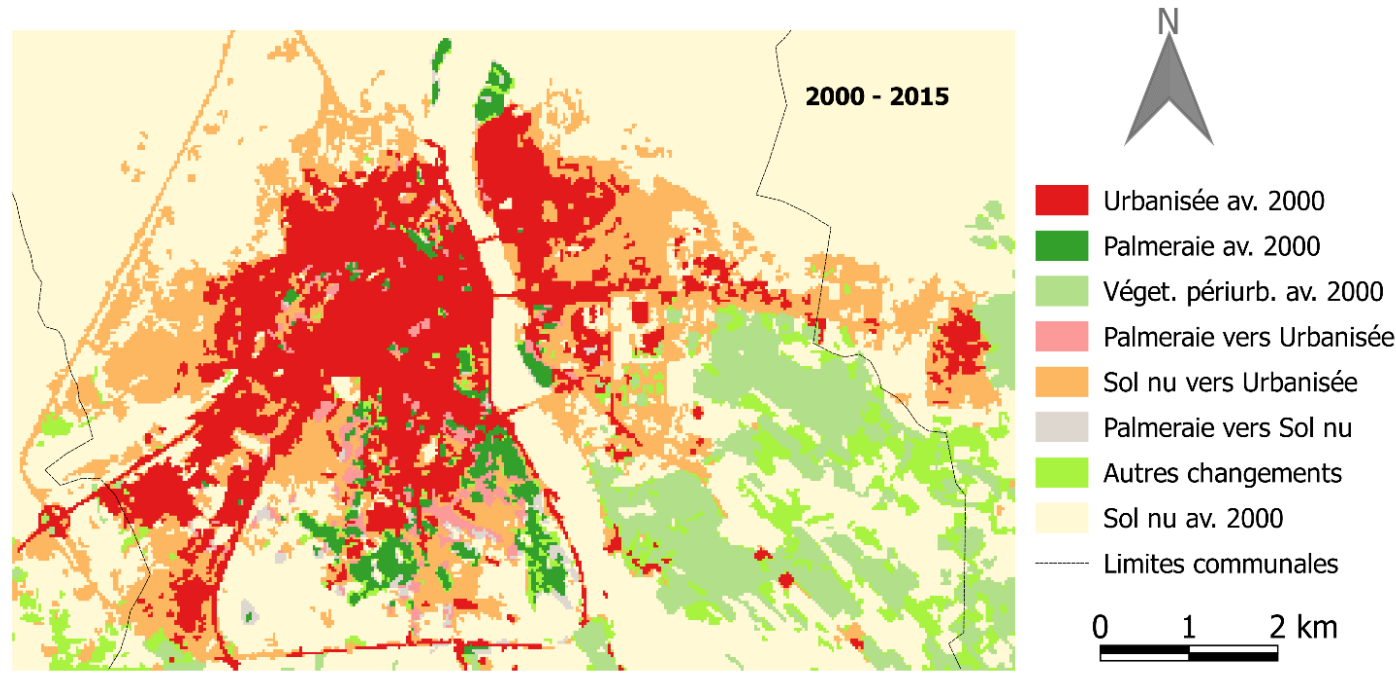

Figure 6 : Changement d'occupation du sol entre 2000 et 2015 


\subsection{Discussion}

En somme, la cartographie du changement spatiotemporel a montré un étalement spatial de la tâche urbaine nettement marqué sur toute la période d'étude, avec néanmoins une plus grande ampleur constatée entre 2000 et 2015. Cet étalement se caractérise par deux traits majeurs :

- d'une part, une conurbation continue étendant la tâche urbaine de Biskra vers les agglomérations périphériques, notamment celle de Chetma à l'est et celle d'El-Hadjeb au sud et au sud-ouest. Les axes de transport ont ainsi influencé cette forme de croissance en conduisant à l'allongement du périmètre urbain ;

- d'autre part, un empiétement progressif des surfaces agricoles constituant la palmeraie. Cette dernière a subi un processus de morcellement et de mitage en conséquence de l'avancée effrénée des surfaces bâties

La détection du changement d'occupation du sol, illustrée dans les figures 03 et 04 , rend compte des répercussions induites par l'étalement du bâti sur la palmeraie par la mise en valeur des surfaces converties par l'urbanisation. La quantification de ces conversions, extraite des rapports descriptifs, révèle que 322,22 ha de surfaces agricoles ont été converties par la classe du bâti entre 1985 et 2015, soit un pourcentage de perte estimé à $47,22 \%$. De plus, les surfaces converties en sol nu sont de l'ordre de 123,03 ha, avec un taux de perte égal à $19,37 \%$. II s'agit là d'une surface dégradée, qui sera éventuellement construite dans l'avenir. Au total, la palmeraie a enregistré une perte globale estimée à $66,56 \%$ de sa superficie, pendant 30 ans. Ce taux de perte montre l'ampleur du mitage induit par l'étalement urbain sur le potentiel oasien représenté par la palmeraie.

\section{Conclusion}

La détection du changement a permis de visualiser et quantifier l'étalement de la ville de Biskra. II ressort que cette ville a enregistré durant ces trois dernières décennies une importante croissance de son tissu urbain, allant jusqu'au-delà de ces limites communales. La portion occupée dans le paysage oasien par la tâche urbaine a considérablement évolué. Elle est passée de $6 \%$ en 1985 à plus de $32 \%$ en 2015 . Globalement, trois directions ont orienté cette expansion : vers le sud, en envahissant la palmeraie; vers le sud-ouest, le long de la route nationale $\mathrm{RN}{ }^{\circ} 46$; vers l'est en conurbation avec l'agglomération de Chetma. En revanche, un recul progressif a caractérisé l'évolution de la palmeraie qui a perdu plus de $62 \%$ de sa part surfacique. La part des surfaces végétales converties par l'urbanisation montre que cette composante écosystémique est en péril face à l'étalement des surfaces bâties.

Cette étude montre l'importance de la télédétection spatiale et des systèmes d'information géographiques pour le suivi et la compréhension de l'étalement rapide des territoires urbains et périurbains, ainsi que l'évaluation de ces impacts sur les écosystèmes avoisinants.

Dans une perspective plus large, cette démarche peut être reproduite pour étudier le phénomène d'urbanisation dans d'autres villes oasiennes et évaluer ainsi leurs modèles de développement urbain. À une échelle plus restreinte, au niveau des quartiers, cette approche peut amener à mesurer et qualifier la végétation urbaine, une composante écologique et bioclimatique essentielle pour une durabilité de la ville saharienne.

\section{Références bibliographiques}

Armand, M. (2016). Images satellitaires et planification des villes du Tiers-Monde. Mondes en développement, 44(176), 168-169. https://doi.org/10.3917/med.176.0169

Ballais, J.-L. (2005). Les villes sahariennes et les risques naturels. In: M. Côte (Éd.), La ville et le désert. Le Bas-Sahara algérien. Paris - Aix-en-Provence: IREMAM-KARTHALA, pp 59-71

Ban, Y., et Yousif, O. (2016). Change Detection Techniques: A Review. In: Y. Ban (Éd.), Multitemporal Remote Sensing Methods and Applications. Cham: Springer International Publishing, pp. 19-43. https://doi.org/10.1007/978-3-319-47037-5_2

Bouzahzah, F. (2015). Dynamique urbaine et nouvelle centralité cas de Biskra -Algérie-. T Univ. Constantine. https://bu.umc.edu.dz/theses/amenagement/BOU6810. pdf

Collet, C., et Caloz, R. (2001). Précis de télédétection: traitements numériques d'images de télédétection. Vol 3. Presses de l'Université du Québec. 386p.

Congalton, R. G. (1991). A review of assessing the accuracy of classifications of remotely sensed data. Remote Sensing of Environment, 37(1), 35-46. https://doi.org/10.1016/0034-4257(91)90048-B

Congalton, R. G., et Green, K. (2008). Assessing the accuracy of remotely sensed data: principles and practices. Boca Raton: CRC press (2nd Ed). 200p.

Congedo, L. (2016). Semi-Automatic Classification Plugin Documentation. Release 6.0.1.1. https://doi.org/10.13140/RG.2.2.29474.02242/1

Coppin, P., Jonckheere, I., Nackaerts, K., Muys, B., et Lambin, E. (2004). Review ArticleDigital change detection methods in ecosystem monitoring: a review. International Journal of Remote Sensing, 25(9), 1565-1596.

https://doi.org/10.1080/0143116031000101675

Du, P., Liu, P., Xia, J., Feng, L., Liu, S., Tan, K., et Cheng, L. (2014). Remote Sensing Image Interpretation for Urban Environment Analysis: Methods, System and Examples. Remote Sensing, 6(10), 9458. https://doi.org/10.3390/rs6109458

Dureau, F., et Weber, C. (1995). Télédétection et systèmes d'information urbains. Paris: Anthropos. 398p.

Farhi, A. (2002). Biskra: de l'oasis à la ville saharienne (Note). Méditerranée, 99, 77-82. https://doi.org/https://doi.org/10.3406/medit.2002.3264

Farhi, B. E., et Hadhaga, F. Z. (2018). Ville oasienne, ville saharienne et ville au sahara: controverse conceptuelle entre rurbanite et contextualite. Courrier $d u$ Savoir, 25, 81-92. http://revues.univbiskra.dz/index.php/cds/article/view/3784

Gamba, P. et Dell'Acqua, F. (2016). Change Detection in Urban Areas: Spatial and Temporal Scales. In: Y. Ban (Éd.), Multitemporal Remote Sensing Methods and Applications. Cham: Springer International Publishing, pp. 19-43. https://doi.org/10.1007/978-3-319-47037-5_3

Girard, M.-C., et Girard, C.-M. (2010). Traitement des 
données de télédétection : Environnement et ressources naturelles. Dunod (2e Éd). 576p.

Hanafi, A., et Alkama, D. (2017). Role of the urban vegetal in improving the thermal comfort of a public place of a contemporary Saharan city. Energy Procedia, 119, 139-152.

https://doi.org/https://doi.org/10.1016/j.egypro.2017.07. 061

Hussain, M., Chen, D., Cheng, A., Wei, H., et Stanley, D. (2013). Change detection from remotely sensed images: From pixel-based to object-based approaches. ISPRS Journal of Photogrammetry and Remote Sensing, 80, 91-106. https://doi.org/10.1016/j.isprsjprs.2013.03.006

Im, J., et Jensen, J. R. (2005). A change detection model based on neighborhood correlation image analysis and decision tree classification. Remote Sensing of Environment, 99(3), 326-340. https://doi.org/10.1016/j.rse.2005.09.008

Jensen, J. R., et Lulla, K. (1987). Introductory digital image processing: A remote sensing perspective. $\begin{array}{lll}\text { Geocarto } & \text { International, } & \text { 2(1), }\end{array}$ https://doi.org/10.1080/10106048709354084

Kouzmine, Y. (2012). Le Sahara algérien. Intégration nationale et développement régional. Paris: L'Harmattan. 344p.

Landis, J. R., et Koch, G. G. (1977). The Measurement of Observer Agreement for Categorical Data. Biometrics, 33(1), 159-174. https://doi.org/10.2307/2529310

Leroux, L., Congedo, L., Bellón, B., Gaetano, R., et Bégué, A. (2018). Land Cover Mapping Using Sentinel2 Images and the Semi-Automatic Classification Plugin: A Northern Burkina Faso Case Study. In QGIS and Applications in Agriculture and Forest (p. 119-151). Hoboken, NJ, USA: John Wiley et Sons, Inc. https://doi.org/10.1002/9781119457107.ch4

Lu, D, Mausel, P., Brondízio, E., et Moran, E. (2004). Change detection techniques. International Journal of Remote Sensing, 25(12), 2365-2401. https://doi.org/10.1080/0143116031000139863

Lu, Dengsheng, et Weng, Q. (2007). A survey of image classification methods and techniques for improving classification performance. International Journal of Remote Sensing, 28(5), 823-870. https://doi.org/10.1080/01431160600746456

Mas, J. F. (2000). Une revue des méthodes et des techniques de télédétection du changement. Canadian Journal of Remote Sensing, 26(4), 349-362. https://doi.org/10.1080/07038992.2000.10874785

Mather, P., et Tso, B. (2016). Classification methods for remotely sensed data (2nd éd.). Boca Raton: CRC press. $376 \mathrm{p}$.

Munafò, M., et Congedo, L. (2017). Measuring and monitoring land cover. In C. Gardi (Éd.), Urban Expansion, Land Cover and Soil Ecosystem Services; Routledge: Abingdon, UK (p. 19-32). London: Routledge.

NASA. (2011). Science Data Users Handbook. 186p. http://landsathandbook.gsfc.nasa.gov/pdfs/Landsat7_H andbook.pdf

Nath, S. S., Mishra, G., Kar, J., Chakraborty, S., et Dey, N. (2014). A survey of image classification methods and techniques. In: Control, Instrumentation, Communication and Computational Technologies (ICCICCT), 2014 International Conference. IEEE, pp 554-557

Phiri, D., et Morgenroth, J. (2017). Developments in Landsat Land Cover Classification Methods: A Review. $\begin{array}{lll}\text { Remote Sensing, } & 9(9), & \end{array}$ https://doi.org/10.3390/rs9090967

Rajendran, P., et Mani, K. (2015). Quantifying the Dynamics of Landscape Patterns in Thiruvananthapuram Corporation Using Open Source GIS Tools. International Journal of Research in Engineering and Applied Sciences, 5(10), 77-87. http://euroasiapub.org/wp-

content/uploads/2016/09/10EASOct-2751-1.pdf

Sezgin, M., et Sankur, B. (2004). Survey over image thresholding techniques and quantitative performance evaluation. Journal of Electronic Imaging, 13(1), 13-20. https://doi.org/10.1117/1.1631315

Singh, A. (1989). Digital Change Detection Techniques Using RemotelySensed Data. International Journal ofRemote Sensing, 10(6), 989-1003. https://doi.org/10.1080/01431168908903939

Skupinski, G., BinhTran, D., et Weber, C. (2009). Les images satellites Spot multi-dates et la métrique spatiale dans l'étude du changement urbain et suburbain-Le cas de la basse vallée de la Bruche (Bas-Rhin, France). Cybergeo: European Journal of Geography. https://doi.org/10.4000/cybergeo.21995

Stehman, S. (1996). Estimating the kappa coefficient and its variance under stratified random sampling. Photogrammetric Engineering and Remote Sensing, 62(4), 401-407

Thériault, M., Sparfel, L., Gourmelon, F., et Le Berre, I. (2011). Modélisation des changements d'occupation et d'utilisation du sol. Cadres formels et exemple d'application. Revue internationale de géomatique, 21(3), 267-295. https://doi.org/10.3166/rig.15.267-295

Tucker, C. J., Grant, D. M., et Dykstra, J. D. (2004). NASA's global orthorectified Landsat data set. Photogrammetric Engineering et Remote Sensing, 70(3), 313-322. https://doi.org/10.14358/PERS.70.3.313

Weng, Y.-C. (2007). Spatiotemporal changes of landscape pattern in response to urbanization. Landscape and Urban Planning, 81(4), 341-353. https://doi.org/10.1016/j.landurbplan.2007.01.009 